\title{
Archivos 2019: Bienvenido OJS, apuesta decidida por la calidad y la visibilidad editorial
}

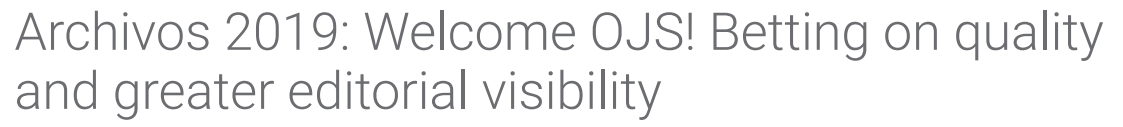

\author{
Laura Fontcuberta ${ }^{1}$ \\ Jordi Delclós ${ }^{1}$ \\ Gullermo García ${ }^{1}$ \\ José Miguel Martínez ${ }^{1}$ \\ José M. Ramada ${ }^{1}$ \\ Elena Ronda ${ }^{1}$
}

${ }^{1}$ Archivos de Prevención de Riesgos Laborales, Barcelona, España

Fechas · Dates

Recibido: 2019.11.09

Aceptado: 2019.12.07

Publicado: 2020.01.15
Correspondencia · Corresponding Author

Elena Ronda

Universidad de Alicante

elena.ronda@ua.es 
Iniciamos esta nota editorial de 2019, con el anuncio de un cambio relevante para los lectores, los diferentes comités, los evaluadores y, claro está, los autores, de Archivos de prevención de Riesgos Laborales y es la adopción de la herramienta de gestión editorial de revistas científicas Open Journal Systems (OJS). Se trata del sistema de gestión de publicaciones científicas más extendido internacionalmente. Esta plataforma, a la que se puede acceder desde este enlace http://archivosdeprevencion.eu/index.php/aprl/login, permite que los usuarios registrados tengan asignado un papel determinado en todo el proceso de gestión, desde el envío del manuscrito hasta la consulta de la gestión, a medida que se va produciendo, sin necesidad de utilizar el correo electrónico como hasta ahora. Así, pues, por ejemplo, los autores podrán conocer, en todo momento, el estado en que se encuentra la revisión de su manuscrito. También los revisores podrán utilizar esta plataforma para el envío y comunicación de sus evaluaciones. La adopción de esta herramienta de gestión responde, por una parte, a nuestra voluntad de adaptación a lo que exige el movimiento de acceso abierto a la ciencia y hacia el cual iniciamos nuestra andadura con la eliminación del acceso restringido a los contenidos científicos de la revista en $2016^{(1)}$. También, por otra parte, es un paso más, hacía nuestro objetivo, de incrementar la visibilidad de la revista como requisito para facilitar su indización en nuevas bases de datos científicas ${ }^{(2)}$.

También durante este año se han producido en cambios en el equipo editorial de la revista. Se incorporan como editores asociados José María Ramada (hasta ahora en el comité editorial) y Guillermo García (coordinador de la sección Archivos Selección). Y, en el caso del Comité Editorial, damos la bienvenida a Santiago Calvet, miembro de la junta de la @SCSL, y a Rocío Villar y Mónica Ubalde. Muchas gracias a todos los que forman parte de este equipo que trabaja en pro de la revista y a los que, a partir de ahora y después de años de colaboración, lo abandonan, aunque continuarán contribuyendo a él desde otros ámbitos.

\section{Gestión de manuscritos: número y tipos de trabajos}

Iniciamos nuestra revisión de este último año de gestión editorial, con la evaluación de determinados aspectos de la revista que nos ayudan a seguir su evolución para mejorar en los aspectos a mejorar. Así, observamos, en la Tabla 1, los manuscritos gestionados este 2019, comparando estos datos con los de los dos años anteriores. No se observan cambios relevantes y, en todas las categorías, los números se mantienen bastante similares a lo largo del trienio. 
Tabla 1: Manuscritos a gestionados en Archivos de Prevención de Riesgos Laborales (2017 a 2019, volúmenes 20, 21 y 22).

\begin{tabular}{|c|c|c|c|}
\hline & 2017 & 2018 & 2019 \\
\hline Aceptados $^{b}$ & 19 & 28 & 22 \\
\hline Rechazados & 18 & 22 & 14 \\
\hline Abandonos & 4 & 2 & 4 \\
\hline En proceso ${ }^{\circ}$ & 1 & 5 & 6 \\
\hline Total & 42 & 57 & 46 \\
\hline
\end{tabular}

a ncluye editoriales, originales, originales breves, artículos especiales, revisiones, artículos de opinión, estudios de casos, notas técnicas y cartas al director hasta el 2 de diciembre de 2019.

b En algún caso, pendientes de publicación en 2020.

- En el momento de elaborarse las Notas Editoriales de los correspondientes años.

Igualmente se analizan los trabajos publicados según su modalidad. En este ámbito, lo que queremos destacar es que, aunque no represente un aumento cualitativo, sí que por primera vez en dos años se han publicado notas técnicas.

Tabla 2: Tipos de artículos a publicados en Archivos de Prevención de Riesgos Laborales (2017 a 2019, volúmenes 20, 21 y 22).

\begin{tabular}{lccc}
\hline & $\mathbf{2 0 1 7}$ & $\mathbf{2 0 1 8}$ & $\mathbf{2 0 1 9}$ \\
\hline Editoriales & 4 & 5 & 4 \\
Notas Editoriales & 1 & 1 & 1 \\
Originales $^{b}$ & 6 & 6 & 7 \\
Revisiones & 1 & 2 & 0 \\
Estudios de casos & 1 & 0 & 2 \\
Notas Técnicas & 0 & 0 & 2 \\
Cartas a la directora $^{\circ}$ & 5 & 3 & 0 \\
Otros $^{c}$ & 4 & 11 & 4 \\
\hline
\end{tabular}

axcluye secciones informativas (Sociedades, Noticias, Archivos Selección, Archivos Evidencia, Archivos Formación)

b Incluye Originales Breves

- Otros: Comunicaciones de la Diada de la @SCSL, Artículos Especiales, Artículos de Opinión, etc.

\section{Tiempos de gestión}

Los tiempos de gestión (Tabla 3) experimentan un ligero crecimiento respecto a 2018 y aunque, no superan los de 2017, seguiremos trabajando para mejorar este indicador. 
Tabla 3: Tiempos de gestión (días) de los manuscritos recibidos en Archivos de Prevención de Riesgos Laborales (2017 a 2019 volúmenes 20, 21 y 22).

\begin{tabular}{lcccccc}
\hline & \multicolumn{2}{c}{$\mathbf{2 0 1 7}$} & \multicolumn{2}{c}{$\mathbf{2 0 1 8}$} & \multicolumn{2}{c}{$\mathbf{2 0 1 9}$} \\
\hline & Mediana & Media & Mediana & Media & Mediana & Media \\
\hline $\begin{array}{l}\text { Recepción- } \\
\text { aceptación }\end{array}$ & 180 & 167 & 102 & 96 & 109 & 117 \\
$\begin{array}{l}\text { Aceptación- } \\
\text { publicación }\end{array}$ & 79 & 74 & 52 & 68 & 67 & 61 \\
$\begin{array}{l}\text { Recepción- } \\
\text { publicación }\end{array}$ & 226 & 242 & 146 & 165 & 175 & 172 \\
\hline
\end{tabular}

a ncluye sólo originales, originales breves, revisiones y estudios de casos

\section{Los autores y contenidos}

En penúltimo lugar, se pueden consultar los datos más relevantes respecto a la información sobre los autores que han publicado sus trabajos en Archivos. Hemos conseguido, volumen tras volumen, que la información relativa a las diferencias por género deje de ser destacable, normalizando la presencia femenina en calidad de primeras autoras, con pequeñas oscilaciones lógicas y esperables en cuanto al número.

Por el contrario, no se ha logrado todavía que la revista sea vista como una oportunidad de comunicación científica para autores de fuera de su área geográfica de influencia más inmediata. Si bien es cierto que hemos conseguido afianzar territorios como Madrid o han vuelto a publicar autores de la Comunidad Valenciana, también lo es, y lo lamentamos, que ha descendido el número de autores de otros países y que hay comunidades autónomas que no tienen presencia en esta tabla, a pesar de que nos consta que sí tenemos lectores de esas comunidades.

No hay sorpresas en cuanto a la filiación de los autores y, una vez más, son mayoría los que trabajan en centros de investigación y universidades. Insistimos, pues, en que aquellos profesionales, cuya experiencia profesional sea de interés en su vertiente más práctica, se animen a publicar sus trabajos en nuestra revista en formatos, como las notas técnicas o los estudios de casos, por ejemplo.

$Y$ ya en último lugar, la Tabla 5 refleja los temas tratados en este volumen 22. Se mantiene cierta diversidad temática, manteniéndose en primer lugar las políticas y estrategias para la prevención. 
Tabla 4: Características de los autoresa (primeros firmantes) que publican en Archivos de Prevención de Riesgos Laborales (2017 a 2019, volúmenes 20, 21 y 22).

2017

2018

2019

\section{Género}

Hombres

Mujeres

$\begin{array}{rrr}6 & 12 & 9 \\ 13 & 14 & 8\end{array}$

Procedencia

\begin{tabular}{lccc}
\hline Andalucía & 0 & 1 & 1 \\
Aragón & 0 & 0 & 0 \\
Asturias & 0 & 0 & 0 \\
Cantabria & 0 & 0 & 0 \\
Castilla y León & 1 & 0 \\
Cataluña & 10 & 13 & 7 \\
C. Valenciana & 3 & 0 & 5 \\
Galicia & 0 & 0 & 0 \\
Islas Baleares & 1 & 0 & 0 \\
Islas Canarias & 0 & 0 & 0 \\
La Rioja & 0 & 2 & 3 \\
Madrid & 1 & 3 & 0 \\
Navarra & 1 & 1 & 0 \\
País Vasco & 0 & 0 & 0 \\
Extremadura & 0 & 0 \\
Otros países & 0 & 0 & 0
\end{tabular}

\section{Institución}

\begin{tabular}{lrrr}
\hline Centros investigación (incluye universidades) & 5 & 11 & 10 \\
Servicios prevención & 0 & 4 & 2 \\
Administración sanitaria & 4 & 7 & 3 \\
Administración laboral & 2 & 0 & 0 \\
Organizaciones sindicales & 0 & 0 & 0 \\
Centros Sanitarios & 5 & 2 & 0 \\
Sociedades científicas & 3 & 2 & 2 \\
\hline
\end{tabular}

a Excluye secciones fijas (Noticias, Archivos Selección, Archivos Evidencia, Archivos Formación)

Tabla 5: Artículos publicados en Archivos de Prevención de Riesgos Laborales (volumen 22, año 2019) según contenidos ${ }^{a}$.

\begin{tabular}{lccccc}
\hline & $\mathbf{N}^{\mathbf{0}}$ & $\mathbf{N}^{\mathbf{2}}$ & $\mathbf{N}^{\mathbf{0}}$ & $\mathbf{N}^{\mathbf{0}} \mathbf{4}$ & Total $\mathbf{2 0 1 9}$ \\
\hline Políticas y estrategias para la prevención & 2 & 3 & 1 & 0 & 6 \\
Evaluación de intervenciones & 1 & 1 & 0 & 0 & 2 \\
Vigilancia de la salud & 0 & 1 & 0 & 0 & 1 \\
$\begin{array}{l}\text { Problemas de salud relacionados con el } \\
\text { trabajo }\end{array}$ & 1 & 0 & 1 & 2 & 4 \\
Evaluación de riesgos & 0 & 0 & 2 & 0 & 2 \\
Enfermedades de origen laboral & 0 & 0 & 0 & 2 & 2 \\
\hline
\end{tabular}

a Excluye secciones fijas (Noticias, Archivos Selección, Archivos Evidencia y Archivos Formación), notas editoriales y comunicaciones de la Diada de la @SCSL 
Queremos utilizar estas líneas que cierran esta nota editorial para expresar nuestra máxima gratitud a todas las personas que contribuyen con su trabajo a que Archivos se siga manteniendo en buen estado de salud: evaluadores externos -reconocidos públicamente en el listado que aparece a continuación de esta nota-, y a los coordinadores de secciones fijas: Guillermo García, Águeda Giráldez, Amàlia Valls, Silvia Royo, Mar Seguí y Consol Serra; a nuestros lectores y autores.

En esta ocasión, queremos mencionar de forma especial a todos los miembros de la Asociación de Medicina del Trabajo de la Comunidad Valenciana por el gran apoyo prestado a la revista. Esta asociación se ha disuelto y ha pasado a integrarse en la Asociación Española de Especialistas en Medicina del Trabajo. Les deseamos lo mejor en esta nueva etapa.

A todos vosotros, pues, feliz y saludable 2020.

\section{Bibliografía}

1. Fontcuberta L, Delclòs J, Martínez JM, León M, Varela P, Ronda E. Archivos 2015: Archivos en abierto. Arch Prev Riesgos Labor 2016; 19: 10-13. doi: 10.12961/ aprl.2016.19.01.2

2. Ronda E, García AM, Schlaghecke JC. Mucho hecho, mucho por hacer. Arch Prev Riesgos Labor. 2015;18:183-4. doi: 10.12961/aprl.2015.18.4.02 


\section{Evaluadores externos para Archivos de Prevención de Riesgos Laborales (2019)}

\begin{tabular}{|c|c|}
\hline Apellidos, Nombre & Institución \\
\hline Albertí, Constança & Institut Català d'Avaluacions Mèdiques i Sanitàries \\
\hline Alguacil, Sara & Mutual Midat Cyclops \\
\hline Arizo-Luque, Vanessa & Universidad Católica de Murcia \\
\hline Benazizi, Ikram & Universidad de Alicante \\
\hline Cabañero, María José & Universidad de Alicante \\
\hline Calvo-Bonacho, Eva & Ibermutua \\
\hline Calvo Cerrada, Beatriz & Institut Català d'Oncologia \\
\hline Climent Rodríguez, José Antonio & Universidad de Huelva \\
\hline De Vicente Abad, M. Ángeles & $\begin{array}{l}\text { Departamento de Investigación e Información. Instituto } \\
\text { Nacional de Seguridad y Salud en el trabajo }\end{array}$ \\
\hline Duran, Xavier & $\begin{array}{l}\text { IMIM - Institut Hospital del Mar d'Investigacions } \\
\text { Mèdiques }\end{array}$ \\
\hline Esteban Buedo, Valentín & $\begin{array}{l}\text { Direcció General de Salut Pública i Addiccions. Conselleria } \\
\text { de Sanitat Universal i Salut Pública de la Generalitat } \\
\text { Valenciana }\end{array}$ \\
\hline García Román, Vicente & Hospital Universitario del Vinalopó, Elche \\
\hline Garcia Shimizu, Patricia & Hospital Marina Baixa, Villajoyosa Alicante \\
\hline Girao, Italo Nicolás & Hospital Universitario Araba, Vitoria/Gasteiz \\
\hline Inglés, Joan & Hospital Universitari Sant Joan \\
\hline Lezaun Goñi, Mercedes & Instituto de Salud Pública y Laboral de Navarra \\
\hline López-Jacob, Ma José & Instituto Sindical de Trabajo, Ambiente y Salud (ISTAS) \\
\hline López López, Juan Carlos & Mutual Midat Cyclops \\
\hline Marhuenda Amorós, Dolores & Universidad Miguel Hernández \\
\hline Martí Fernández, Francesc & Mutual Midat Cyclops \\
\hline Martí Margarit, Anna & Cualtis \\
\hline Martinez-Tur, Vicente & Universidad de Valencia \\
\hline Palmer, Rafael & $\begin{array}{l}\text { Direcció General de Treball, Economia Social i Salut } \\
\text { Laboral. Conselleria de Treball, Comerç i Indústria. Govern } \\
\text { Balear }\end{array}$ \\
\hline Pascual del Pobil Ferré, Mª Amparo & $\begin{array}{l}\text { Conselleria de Sanitat Universal i Salut Pública. } \\
\text { Generalitat de la Comunitat Valenciana }\end{array}$ \\
\hline Rimbau Gilabert, Eva & Universitat Oberta de Catalunya \\
\hline Rodríguez Blanes, Gloria Ma & $\begin{array}{l}\text { Centro de Salud Pública de Alcoy. Consellería de Sanidad } \\
\text { Universal y Salud Pública, Generalitat Valenciana }\end{array}$ \\
\hline Salcedo Beltrán, Carmen & Universidad de Valencia \\
\hline Sanjuan Quiles, Angela & Universidad de Alicante \\
\hline
\end{tabular}




\begin{tabular}{ll}
\hline Apellidos, Nombre & Institución \\
\hline Sol Vidiella, Josep & Institut Català de la Salut. Hospital de Tortosa Verge de la \\
& Cinta i Servei d'Atenció Primària Terres de l'Ebre \\
Soler Font, Mercè & Centre d'Investigació en Salut Laboral (CISAL), Universitat \\
& Pompeu Fabra \\
Terradas Robledo, M. Roser & IMIM Parc de Salut Mar i Cisal (Centre d'Investigació en \\
& Salut Laboral), UPF \\
Valencia Martín, José Lorenzo & Servicio de Medicina Preventiva y Salud Pública. Hospital \\
& Universitario Ramón y Cajal \\
Vaquero, Manuel & Universidad de Córdoba \\
Velarde Collado, José María & Unitat de VIH i ITS de l'Hospital Universitari de Bellvitge \\
Vicente Herrero, Ma Teófila & Servicio de Prevención Correos. Grupo de Investigación \\
& en Medicina del Trabajo (GIMT) \\
Villar Vinuesa, Rocío & IMIM Parc de Salut Mar i Cisal (Centre d'Investigació en \\
Zhao, Guanlan & Salut Laboral), UPF \\
Zapater, Reyes & Universidad de Alicante \\
& Servicio de prevención de Conselleria de Sanidad \\
& Universal y Salud Pública, Generalitat Valenciana \\
\hline
\end{tabular}

\section{¡Con nuestro reconocimiento y gratitud!}

